\title{
EL BUQUE SUR: UN ENTIERRO PRIMARIO MÚLTIPLE DE 2300 AÑOS EN LA COSTA DEL GOLFO SAN MATÍAS, RÍO NEGRO, ARGENTINA.
}

\author{
SOLANA GARCÍA GURAIEB", CAROLINA I. MARIANO** Y CRISTIAN M. FAVIER DUBOIS"***
}

\begin{abstract}
RESUMEN
Se presentan los resultados de distintos aspectos bioarqueológicos del entierro primario de cuatro individuos adultos recuperados en el sitio El Buque Sur (Río Negro), fechado en ca. 2300 años AP. Se incluyen el análisis del contexto mortuorio, la composición sexual y etaria del entierro y los análisis tafonómicos, paleopatológicos e isotópicos de los restos óseos. El hallazgo se realizó durante las obras de excavación de una cisterna, por los que la primera etapa de la excavación fue realizada por el personal local de Bomberos. Posteriormente, se dio intervención al equipo de investigación arqueológica, que realizó el rescate del cuarto individuo completo. La integridad de la muestra se vio afectada por la mezcla y pérdida de algunos elementos óseos. No obstante, se pudo reconstruir la mayor parte de la misma y obtener importante información bioarqueológica. Los resultados obtenidos se discuten en el marco de la evidencia bioarqueológica disponible para el área del golfo San Matías.
\end{abstract}

PALABRAS CLAVES: cazadores-recolectores costeros, prácticas mortuorias, paleopatologías, tafonomía, dieta.

\section{EL BUQUE SUR: A MULTIPLE PRIMARY BURIAL OF 2300 YEARS BP IN THE SAN MATIAS GULF COAST (RÍO NEGRO, ARGENTINA).}

\begin{abstract}
Different aspects of the bioarchaeological study of the burial site El Buque Sur (Río Negro, Argentina), are presented. The site is a primary multiple burial of four adult individuals dating ca. 2300 years BP. Mortuary context, sex and age composition as well as paleopathological, taphonomic and isotopic analyses are included in this study. The burial was found during the excavation of a pit for a cistern on the beach, which led to the first part of the recovery to be undertaken by local fire-fighters. Afterwards, the archaeological research team was summoned and was able to perform the archaeological excavation of the fourth skeleton. The non-archaeological recovery negatively affected their completeness and caused
\end{abstract}


the mixture of the bones. Notwithstanding, the reconstruction of most of the first three individuals made it possible to obtain valuable data. Results obtained are presented here and discussed in relation to the bioarchaeological data available for the San Matías Gulf coast.

KEYWORDS: coastal hunter gatherers, primary burial, taphonomy, paleopathology, diet.

\section{INTRODUCCIÓN}

Desde el año 2003 vienen llevándose a cabo en la costa del golfo San Matías, investigaciones arqueológicas cuyo objetivo es conocer la dinámica de uso del espacio costero por parte de las poblaciones cazadoras-recolectoras que habitaron el área en el pasado (Favier Dubois et al. 2006; Favier Dubois et al. 2009). Los resultados obtenidos muestran una importante diferencia en la densidad y distribución del registro arqueológico entre la costa norte y la costa oeste del golfo San Matías (Fig.1) que parece estar relacionada con la desigual configuración litoral y la base de recursos disponibles (Borella y Favier Dubois 2005; Favier Dubois et al. 2006). De este modo, la mayor abundancia de recursos críticos, tales como el agua dulce, presentes en la costa septentrional del golfo San Matías, podría dar cuenta de la mayor densidad, abundancia y diversidad del registro arqueológico en este sector (Favier Dubois et al. 2006; Favier Dubois et al. 2009). En este marco, el análisis de diversos aspectos del registro bioarqueológico presenta un importante potencial para la comprensión de la dinámica de ocupación del área y ha constituido uno de los objetivos destacados de las investigaciones en curso (Borella et al. 2007, Favier Dubois et al. 2007, 2009, Mariano 2009). Estos trabajos han ratificado las tendencias arqueológicas generales, mostrando que el registro bioarqueológico también presenta una distribución diferencial entre la costa norte y la costa oeste (Favier Dubois et al. 2007; Mariano 2009). Asimismo, los estudios isotópicos realizados sobre restos humanos han permitido observar tendencias temporales en la intensidad de uso de los recursos marinos (Favier Dubois et al. 2009). Estos trabajos cobran particular relevancia para el área de estudio, ya que hasta el momento se contaba con escasos antecedentes de estudios bioarqueológicos previos (e.g. Deodat en Bórmida 1953-1954; Sánchez Albornoz 1967).

En ese contexto, este trabajo tiene por objeto dar a conocer uno de los entierros más antiguos y completos recuperados en el área, denominado $\mathrm{El}$ Buque Sur. Se trata de un entierro primario múltiple, cuyo hallazgo fue denunciado por obreros que se encontraban realizando una construcción en la zona, por lo cual parte de su excavación fue realizada por personal no especializado, que intervino antes de que se notificara al equipo de investigación arqueológica. Se presentan las tareas realizadas para evaluar y mitigar el daño que la manipulación no arqueológica ocasionó en los restos óseos y se dan a conocer los resultados del estudio bioarqueológico realizado, que incluye el análisis del contexto mortuorio, su cronología y composición sexual y etaria, así como estudios tafonómicos, paleopatológicos y paleodietarios. Estos resultados se discuten en relación con la información arqueológica y bioarqueológica disponibles para el área, particularmente la referida a las tendencias detectadas en el uso del espacio y los recursos a lo largo del Holoceno tardío (Favier Dubois et al. 2009).

\section{EL HALLAZGO}

El entierro de El Buque Sur - BS- (GPS: $40^{\circ}$ 52 ' 5.8" de latitud sur y 65 $07^{\prime} 28.9$ " de longitud oeste) se ubica en el extremo septentrional de la costa oeste del golfo San Matías, $6 \mathrm{~km}$ al sur de la localidad de Las Grutas. El hallazgo se produjo en septiembre de 2005 en un médano costero. Ante la aparición de los restos se dio aviso a la Policía de Las Grutas, a la Fiscalía y a los Bomberos, quienes efectuaron la extracción a pala de numerosos huesos correspondientes a los primeros tres individuos, ocasionando la mezcla y el daño de los mismos. Gracias al pedido expreso de representantes de la comunidad Mapuche de San Antonio Oeste y a la difusión pública del hallazgo, el equipo de investigación arqueológica pudo contactarse con las autoridades locales y realizar el rescate del cuarto individuo y recuperar los elementos y fragmentos óseos correspondientes a los primeros tres esqueletos extraídos. 


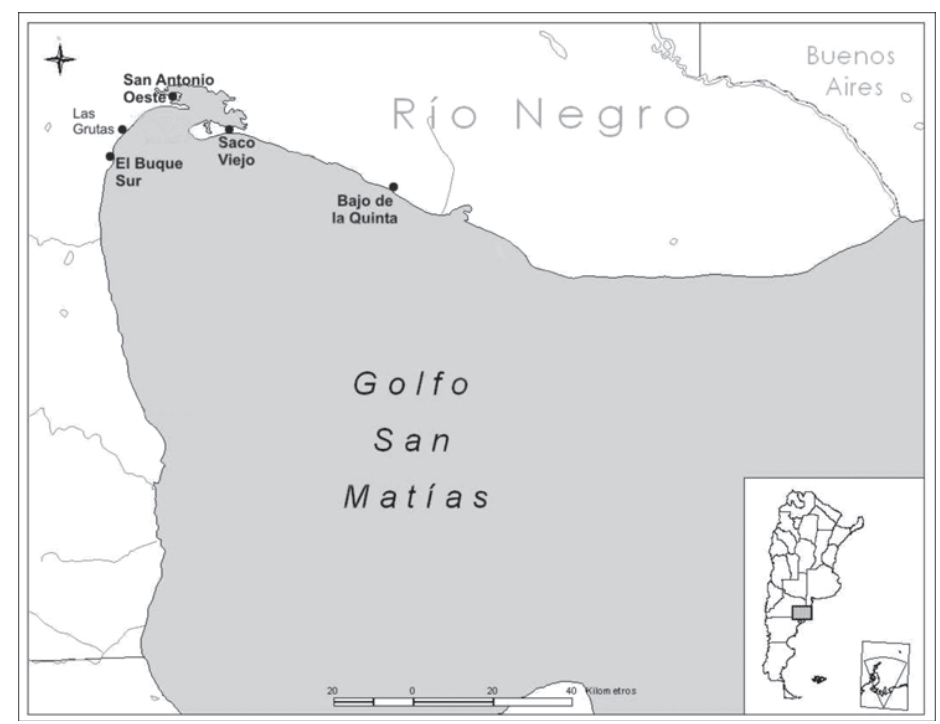

Fig. 1. Mapa del Gofo San Matías con la ubicación del entierro El Buque Sur.

\section{METODOLOGÍA DE ANÁLISIS}

En el estudio del entierro de El Buque Sur se consideraron los siguientes aspectos: contexto mortuorio, análisis tafonómico, composición sexual y etaria del entierro, estudio paleopatológico y análisis paleodietarios.

En la caracterización del contexto mortuorio se tuvo en cuenta el contexto geomorfológico de depositación, la posición de los cuerpos y la presencia/ausencia de materiales culturales asociados y estructuras funerarias. Para los primeros tres individuos esta información se obtuvo del personal de bomberos que los exhumó, o bien fue inferida a partir de ciertas características tafonómicas de los elementos óseos (ver infra). Para establecer la cronología del entierro se realizaron dos fechados radiocarbónicos por AMS sobre los terceros molares de dos de los individuos.

Dadas las características de este hallazgo, la primera etapa del análisis se centró en tareas de acondicionamiento y separación de los huesos de los primeros tres esqueletos recuperados. Para ello se consideraron las dimensiones, lateralidad y características morfológicas y tafonómicas de los elementos óseos (e.g. tinciones o sustancias adheridas como carbonato de calcio). Posteriormente se continuaron los estudios tafonómicos con el doble fin de analizar el proceso de formación del entierro y medir el impacto que la recuperación no arqueológica de los tres primeros individuos tuvo sobre la integridad de la muestra. Se relevaron la presencia de fracturas postmortem, la pérdida ósea post-depositacional, la presencia de tinciones y sustancias adheridas, la impronta de raíces, las marcas de roedores y carnívoros y la meteorización. Asimismo se evaluó el grado de completitud y de fragmentación de los cuatro esqueletos a través del cálculo de un índice de completitud anatómica y el índice de fragmentación (IF); este último empleando la propuesta de Mondini (2003).

La determinación del sexo de cada individuo se realizó a partir de observaciones morfológicas del cráneo y la pelvis (recopilados por Buikstra y Ubelaker 1994). La estimación de la edad se efectuó a través de la consideración de múltiples indicadores, entre los que se privilegiaron los cambios morfológicos de la sínfisis púbica (Brooks y Suchey 1990) y de la superficie auricular y retroauricular del ilion (Lovejoy et al. 1985).

El estudio de las alteraciones patológicas se efectuó de forma macroscópica y en casos particulares se tomaron radiografías para contribuir a la clasificación etiológica de las lesiones observadas. En primer lugar, se registraron diversos indicadores inespecíficos de estrés, tales como hipoplasias de esmalte dental (sensu Hillson 1996), hiperostosis porótica y criba orbitaria (sensu Mensforth 1991), reacciones periósticas (sensu Weston 2008), artropatías (Buisktra y Ubelaker 1994; Rogers y 
Waldron 1995) y traumas (Ortner 2003). También se relevaron indicadores de salud bucal tales como desgaste dental, caries, abscesos, pérdida de dientes antemortem y enfermedad periodontal (siguiendo los criterios de Hillson 1996; Clarke y Hirsch 1991). En segundo lugar, se efectuó un completo relevamiento de lesiones óseas, que permitiera detectar otro tipo de patologías. Para ello se registraron el tipo de lesión, severidad, grado de remodelación, dimensiones y localización de alteraciones en cada elemento óseo. A nivel del individuo, se consignaron los elementos afectados y la presencia de lesiones similares en el resto del esqueleto. De esta forma se garantizó una instancia descriptiva, previa a la discusión de diagnósticos diferenciales y la clasificación de las lesiones en categorías etiológicas amplias (Lovell 2000).

Por último, se incluyeron en los resultados y la discusión general los valores isotópicos de $\delta^{13} \mathrm{C}$ y $\delta^{15} \mathrm{~N}$ obtenidos sobre colágeno, relacionándolos con los datos de la ecología isotópica local (Favier Dubois et al. 2009).

\section{RESULTADOS}

Contexto Mortuorio: El Buque Sur es un entierro primario múltiple, sin estructura funeraria evidente, situado en un médano actualmente edafizado. La tinción oscura en la mayoría de los elementos del individuo BS1 permite suponer que se ubicaba por encima de los otros tres individuos, en lo que correspondería al horizonte A del suelo desarrollado sobre los depósitos de arena. La distribución de frecuencias de las tinciones y marcas de raíces en los restos de los individuos BS2 y BS3 sugerirían que BS2 se encontraba estratigráficamente por encima del individuo BS3 (ver infra). El cuarto individuo, BS4, fue hallado a 1,50 metros de profundidad en lo que habría sido la base del entierro. Se encontraba en posición decúbito lateral derecha, flexionado, con la cabeza sobre el lateral derecho y hacia abajo (Fig. 2). La orientación del eje mayor del cuerpo de BS4 era NNO (cabeza)- SSE (pies). No se encontraron materiales culturales asociados a ninguno de los individuos.

1 La metodología general empleada en este trabajo ha sido desarrollada detalladamente en trabajos previos (García Guraieb 2006), excepto para el caso de las artropatías, por lo que al presentar esos resultados se explicita cómo se realizaron las cuantificaciones.

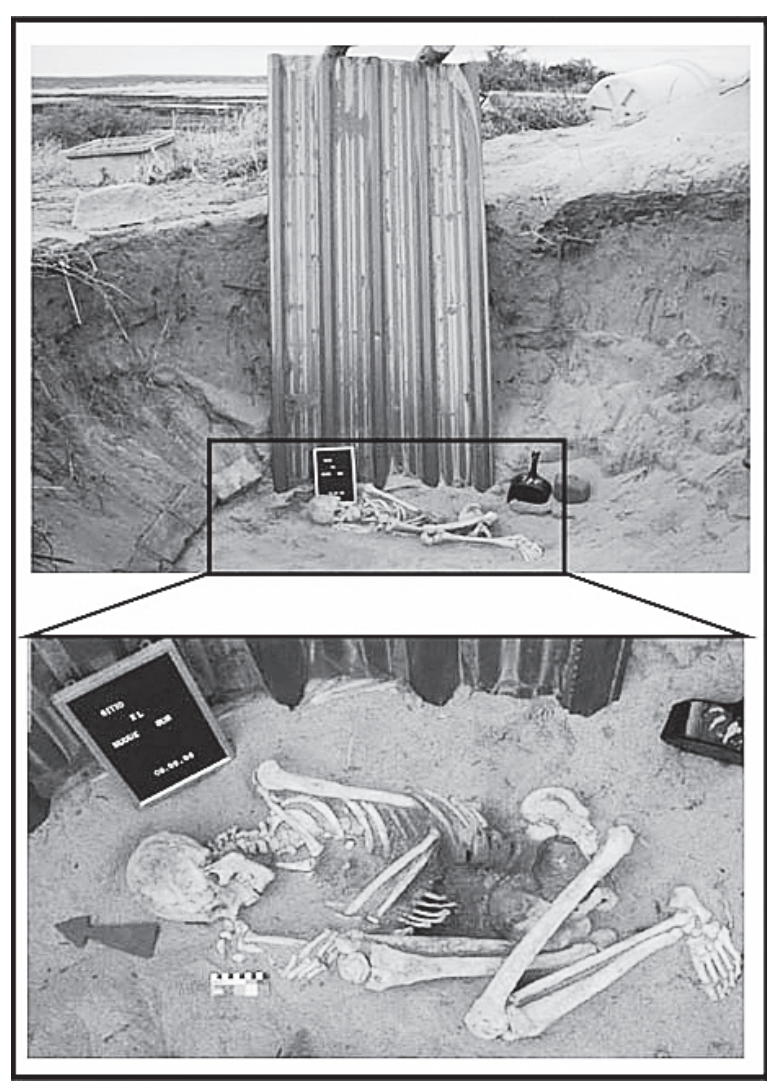

Fig. 2. Individuo BS4 in situ: ubicación estratigráfica (sup.) y detalle de la posición anatómica (inf.).

En la tabla 1 se presentan las edades radiocarbónicas obtenidas de los individuos BS1 y BS4, el más superficial y el más profundamente ubicado en el entierro, respectivamente. La superposición prácticamente a un sigma de los fechados radiocarbónicos, la proximidad espacial de los esqueletos dentro del entierro y la ausencia de una estructura funeraria visible y perdurable en el tiempo, que permitiera la reubicación del entierro en un ambiente dinámico como el de dunas costeras (e.g. una acumulación de rocas), permiten suponer que se trata de un solo evento de depositación de los cuerpos o de eventos separados por lapsos cortos de tiempo.

Reconstrucción de los individuos y composición sexual y etaria del entierro: En total se recuperaron 720 restos óseos, de los cuales el 70\% pudo identificarse anatómica (511 especímenes que representan 472 elementos). Entre los restos que no pudieron ser asignados a un individuo en particular 
Tabla 1. Fechados radiocarbónicos obtenidos para los dos esqueletos de El Buque Sur.

\begin{tabular}{|c|c|c|c|c|}
\hline Individuo & Muestra & $\begin{array}{c}\text { Fechado AMS sin } \\
\text { calibrar (en años AP) }\end{array}$ & $\begin{array}{c}\text { Fechado calibrado* } \\
\text { (en años AP) }\end{array}$ & Código de lab. \\
\hline BS1 & molar & $2195 \pm 49$ & $2009-2299$ & AA70720 \\
\hline BS4 & molar & $2300 \pm 49$ & $2159-2336$ & AA70719 \\
\hline
\end{tabular}

"Fechas calibradas con el programa CALIB REV 5.0.2 (1986-2005) (Stuiver y Reimer

1993), a un sigma, Curva para el Hemisferio Sur (Favier Dubois et al. 2009).

se encuentran fragmentos de costillas (134), de vértebras y sacro (40), huesos de las manos (23) y de los pies (12). La mayor parte de los elementos óseos del conjunto de huesos "mezclados" pudo ser asignada a alguno de los tres individuos, lo que permitió determinar la composición sexual y etaria del entierro. El mismo se compone de cuatro individuos adultos: dos femeninos y dos masculinos. En la tabla 3 se presenta un resumen de los elementos óseos representados en cada caso, el sexo y la edad estimados para cada uno.

Análisis tafonómico: La tabla 3 presenta los resultados del análisis tafonómico efectuado. Los índices de completitud anatómica y fragmentación de cada esqueleto, evidencian que el individuo BS4 muestra el mayor grado de completitud y el menor de fragmentación, seguido por los individuos BS1, BS3 y BS2. Los procesos que más afectaron los huesos de los primeros tres individuos fueron la pérdida ósea postdepositacional y las fracturas postmortem. El porcentaje de ambas variables es marcadamente menor en el individuo BS4. Con respecto a las sustancias adheridas, los elementos óseos de los cuatro esqueletos estaban cubiertos por una fina capa de carbonato de calcio. Especialmente en los individuos BS1 y BS2 se registró una tinción distribuida en forma de grandes manchas oscuras, cuyo origen aún no pudo determinarse con claridad, pero podría tratarse de óxido de manganeso y/o

Tabla 2. Sexo, edad y elementos óseos representados en cada individuo de El Buque Sur.

\begin{tabular}{|c|c|c|l|}
\hline Indiv. & Sexo & $\begin{array}{c}\text { Edad } \\
\text { (en años) }\end{array}$ & \multicolumn{1}{c|}{ Elementos representados } \\
\hline BS1 & M & $40-44.9$ & $\begin{array}{l}\text { Cráneo y mandíbula, cinturas escapulares, fragmentos vertebrales y del sacro, miembros } \\
\text { superiores e inferiores, coxales, huesos de manos y pies. }\end{array}$ \\
\hline BS2 & F & $45-59.9$ & $\begin{array}{l}\text { Cráneo y mandíbula, cinturas escapulares, atlas, lumbares y fragmentos de sacro, miembros } \\
\text { superiores e inferiores, coxales, huesos de manos y pies. }\end{array}$ \\
\hline BS3 & F & $40-49.9$ & $\begin{array}{l}\text { Cráneo y mandíbula, cinturas escapulares, cervicales, dorsales 1 y 12, lumbares y fragmentos de } \\
\text { sacro, miembros superiores (excepto radio izquierdo) e inferiores, coxales, huesos de manos y pies. }\end{array}$ \\
\hline BS4 & M & $25-40$ & Todos, excepto falanges del pie derecho. \\
\hline
\end{tabular}

Tabla 3. Resultados de las variables e índices tafonómicos analizados por individuo.

\begin{tabular}{|c|c|c|c|c|c|c|c|c|c|}
\hline Ind. & NME & $\begin{array}{c}\text { Índice de } \\
\text { Completitud } \\
\text { anatómica } \\
\text { (NME /NEE) }\end{array}$ & NISP & $\begin{array}{c}\text { Índice de } \\
\text { fragmen. } \\
\text { (NME/NISP) }\end{array}$ & $\begin{array}{c}\text { \% de NME } \\
\text { con fracturas } \\
\text { postmortem }\end{array}$ & $\begin{array}{c}\text { \% de NME } \\
\text { con pérdida } \\
\text { ósea post- } \\
\text { deposit. }\end{array}$ & $\begin{array}{c}\text { \% de NME } \\
\text { con } \\
\text { carbonato }\end{array}$ & $\begin{array}{c}\% \text { de NME } \\
\text { con sustancia } \\
\text { oscura } \\
\text { adherida }\end{array}$ & $\begin{array}{c}\text { \% de NME } \\
\text { con marcas } \\
\text { de raíces }\end{array}$ \\
\hline BS1 & 114 & $\begin{array}{c}0,55 \\
(114 / 208)\end{array}$ & 120 & $\begin{array}{c}0,95 \\
(114 / 120)\end{array}$ & $\begin{array}{c}8,77 \\
(10 / 114)\end{array}$ & $\begin{array}{c}52,63 \\
(60 / 114)\end{array}$ & $\begin{array}{c}100 \\
(114 / 114)\end{array}$ & $\begin{array}{c}54,39 \\
(62 / 114)\end{array}$ & $\begin{array}{c}71,05 \\
(81 / 114)\end{array}$ \\
\hline BS2 & 77 & $\begin{array}{c}0,37 \\
(77 / 208)\end{array}$ & 95 & $\begin{array}{c}0,81 \\
(77 / 95)\end{array}$ & $\begin{array}{c}12,99 \\
(10 / 77)\end{array}$ & $\begin{array}{c}54,55 \\
(42 / 77)\end{array}$ & $\begin{array}{c}100 \\
(77 / 77)\end{array}$ & $\begin{array}{c}10,39 \\
(8 / 77)\end{array}$ & $\begin{array}{c}58,44 \\
(45 / 77)\end{array}$ \\
\hline BS3 & 95 & $\begin{array}{c}0,45 \\
(95 / 208)\end{array}$ & 108 & $\begin{array}{c}0,88 \\
(95 / 108)\end{array}$ & $\begin{array}{c}15,79 \\
(15 / 95)\end{array}$ & $\begin{array}{c}62,11 \\
(59 / 95)\end{array}$ & $\begin{array}{c}100 \\
(95 / 95)\end{array}$ & $\begin{array}{c}1,05 \\
(1 / 95)\end{array}$ & $\begin{array}{c}45,26 \\
(43 / 95)\end{array}$ \\
\hline BS4 & 186 & $\begin{array}{c}0,89 \\
(186 / 208)\end{array}$ & 188 & $(186 / 188)$ & $(9 / 186)$ & $\begin{array}{c}26,88 \\
(50 / 186)\end{array}$ & $\begin{array}{c}100 \\
(186 / 186)\end{array}$ & $\begin{array}{c}1,08 \\
(2 / 186)\end{array}$ & $\begin{array}{c}19,89 \\
(37 / 186)\end{array}$ \\
\hline
\end{tabular}

NME: número mínimo de elementos recuperados por individuo; NEE: número de elementos esperados por individuo. (=208 en promedio para un individuo adulto); NME: número de elementos recuperados y asignados a uno de los 4 individuos. 
Tabla. 4. Prevalencias individuales y muestrales de los indicadores de salud bucal.

\begin{tabular}{|c|c|c|c|c|c|c|c|c|c|c|}
\hline \multirow{2}{*}{$\begin{array}{c}\text { Individuo } \\
\text { (Sexo, edad) }\end{array}$} & \multirow{2}{*}{$\begin{array}{c}\text { Alvé. } \\
\text { Obs. }\end{array}$} & \multirow{2}{*}{$\begin{array}{c}\text { Dient. } \\
\text { Obs. }\end{array}$} & \multicolumn{2}{|c|}{$\begin{array}{c}\text { Pérdidas } \\
\text { Postmortem }\end{array}$} & \multicolumn{2}{c|}{$\begin{array}{c}\text { Pérdidas } \\
\text { Antemortem }\end{array}$} & \multicolumn{2}{|c|}{ Abscesos } & \multicolumn{2}{|c|}{ Caries } \\
\cline { 5 - 12 } & & & $\mathrm{N}$ & $\%$ & $\mathrm{~N}$ & $\%$ & $\mathrm{~N}$ & $\%$ & $\mathrm{~N}$ & $\%$ \\
\hline BS1 (M, 40-44,9) & 32 & 16 & 7 & 21,9 & 9 & 28,1 & 3 & 9,4 & 0 & 0 \\
\hline BS2 (F, 45-59.9) & 32 & 29 & 3 & 9,4 & 0 & 0 & 2 & 6,3 & 2 & 6,9 \\
\hline BS3 (F, 40-49.9 & 32 & 29 & 1 & 3,1 & 2 & 6,3 & 3 & 9,4 & 0 & 0 \\
\hline BS4 (M, 25-40) & 32 & 31 & 0 & 0 & 1 & 3,1 & 3 & 9,4 & 0 & 0 \\
\hline TOTAL & 128 & 105 & 11 & 8,6 & 12 & 9,3 & 11 & 8,6 & 2 & 1,9 \\
\hline
\end{tabular}

ácidos húmicos. La acción de raíces sólo afectó la capa de carbonato que cubría los elementos y decrece su acción desde el individuo BS1 al BS4. La presencia de estas características contribuyó a discriminar los restos de los tres individuos mezclados y la distribución de sus frecuencias ratificaría la inferencia sobre su ubicación estratigráfica en el entierro. Sólo tres elementos óseos mostraron marcas de roedores y ninguno de carnívoros. Tampoco hay evidencias de meteorización ósea ni de huellas antrópicas distintas a las causadas por la excavación no arqueológica (e.g. como huellas de corte, raspado, etc.). En suma, los estudios tafonómicos sugieren que el entierro sólo habría sufrido modificaciones postdepositacionales vinculadas a las condiciones de depositación en un ambiente de médano edafizado (e.g. carbonato, tinciones, improntas de radículas) que podrían haber contribuido a la pérdida de colágeno de los huesos y a incrementar su fragilidad, pero que no habrían ocasionado desarticulaciones de importancia.

Estudio paleopatológico: Las prevalencias de los indicadores de salud bucal a nivel muestral $e$ individual se indican en la tabla 4 . Se observa muy baja frecuencia de caries (menor al 2\% de piezas observadas). Las mismas corresponden al individuo BS2, que mostró una pequeña caries de cuello sobre el sector vestibular del canino superior derecho y una caries incipiente en el fondo de uno de los surcos del tercer molar superior izquierdo. Se advierten también frecuencias relativamente bajas de abscesos y pérdidas ante y postmortem. El individuo BS1 contribuye con las mayores frecuencias de pérdidas ante y postmortem a esta cifra general, mientras que la distribución de las frecuencias de abscesos es pareja entre los cuatro individuos. Se observaron abscesos periapicales y complejos. La mayoría de los mismos se detectó en alvéolos con piezas in situ, con altos grados de desgaste que habría provocado la exposición e infección de la cavidad pulpar. El individuo BS1 fue el único que mostró evidencias óseas de enfermedad periodontal en su maxilar superior. Junto con los abscesos registrados, ambas condiciones podrían dar cuenta de la relativamente alta proporción de pérdidas dentales antemortem en este individuo. Por último, los cuatro individuos muestran altos grados de desgaste dental, consistentes con la edad estimada para ellos, en su mayoría mayores a cuarenta años de acuerdo con los indicadores pélvicos considerados.

Debido al alto grado de desgaste coronario y a la proporción de piezas perdidas, no fue posible evaluar la presencia de hipoplasias lineales de esmalte dental. Tampoco se observaron evidencias de hiperostosis porótica, criba orbitaria ni reacciones periósticas en ninguno de los individuos. Asimismo, exceptuando los abscesos y caries descriptos, no se hallaron evidencias de procesos infecciosos en el conjunto.

Con respecto a las artropatías, se relevaron 10 regiones articulares postcraneales (Tabla 5) y las articulaciones vertebrales (cuerpos y facetas articulares) para identificar la presencia de osteoartritis (OA) de las articulaciones sinoviales y espondilosis deformante en los cuerpos vertebrales. En el relevamiento de OA, no se registraron casos de eburnación -su rasgo patognómico- por lo que para identificar la condición en la muestra se empleó como criterio la ocurrencia conjunta de al menos dos de las siguientes alteraciones: osteofitos marginales (OM) o superficiales (OS), porosidad (P) y alteración morfológica del contorno de la superficie articular (AC) (Rogers y Waldron 1995). Los resultados obtenidos (Tabla 5) muestran la presencia de OA en todos los individuos en al menos una articulación, aunque no se observan recurrencias de las regiones articulares afectadas entre los individuos. Cabe destacar que el 
Tabla 5. Alteraciones articulares postcraneales (no columnares).

\begin{tabular}{|c|c|c|c|c|c|c|c|c|c|}
\hline \multirow{2}{*}{\multicolumn{2}{|c|}{$\begin{array}{l}\text { Regiones articulares/ } \\
\text { superficies articulares }\end{array}$}} & \multicolumn{2}{|c|}{ BS1 } & \multicolumn{2}{|c|}{ BS2 } & \multicolumn{2}{|c|}{ BS3 } & \multicolumn{2}{|c|}{ BS4 } \\
\hline & & Izq & Der & Izq & Der & Izq & Der & Izq & Der \\
\hline \multicolumn{2}{|c|}{ Acrom.-clavicular } & 0 & $\mathrm{OA}$ & $0^{*}$ & 0 & $0^{*}$ & 0 & 0 & 0 \\
\hline \multicolumn{2}{|c|}{ Esterno-clavicular } & A & A & $0^{*}$ & 0 & $0^{*}$ & $0^{*}$ & 0 & $0^{*}$ \\
\hline \multirow[t]{2}{*}{ Hombro } & Húmero $\mathrm{px}$ & 0 & $0^{*}$ & $\mathrm{OA}$ & $0^{*}$ & 0 & $\mathrm{~A}$ & 0 & 0 \\
\hline & Glenoid & A & 0 & A & 0 & 0 & A & 0 & 0 \\
\hline \multirow[t]{3}{*}{ Codo } & Húmero ds & 0 & 0 & $0^{*}$ & OA & $\mathrm{OM}$ & $\mathrm{OM}$ & 0 & $\mathrm{OM}$ \\
\hline & Cúbito ds & $\mathrm{OM}, \mathrm{OS}$ & $\mathrm{OM}, \mathrm{OS}$ & $\mathrm{OM}$ & OM & $0^{*}$ & $\mathrm{OM}, \mathrm{OS}$ & $\mathrm{OM}, \mathrm{OS}$ & $\mathrm{OA}$ \\
\hline & Radio ds & 0 & 0 & 0 & 0 & $0^{*}$ & & 0 & $\mathrm{P}$ \\
\hline \multirow[t]{2}{*}{ Muñeca } & Cúbito ds & $0^{*}$ & 0 & $0^{*}$ & 0 & $0^{*}$ & 0 & $\mathrm{OA}$ & 0 \\
\hline & Radio ds & 0 & 0 & 0 & 0 & $0^{*}$ & & $\mathrm{OA}$ & 0 \\
\hline \multicolumn{2}{|c|}{ Mano } & $0^{*}$ & $0^{*}$ & $0^{*}$ & $0^{*}$ & $0^{*}$ & $0^{*}$ & $\mathrm{OA}$ & 0 \\
\hline \multirow[t]{2}{*}{ Cadera } & Fémur px & 0 & 0 & 0 & 0 & A & OA & 0 & 0 \\
\hline & Acetábulo & OM & $\mathrm{OM}$ & $0^{*}$ & 0 & 0 & 0 & OM & $\mathrm{OM}$ \\
\hline \multirow[t]{3}{*}{ Rodilla } & Fémur ds & 0 & 0 & $\mathrm{P}$ & $0^{*}$ & $0^{*}$ & $0^{*}$ & $\mathrm{P}$ & $P$ \\
\hline & Rótula & A & A & 0 & 0 & $0^{*}$ & $0^{*}$ & 0 & 0 \\
\hline & Tibia px & $0^{*}$ & $0^{*}$ & OS & $0^{*}$ & $\mathrm{OA}$ & $\mathrm{OM}, \mathrm{OS}$ & 0 & 0 \\
\hline \multirow[t]{2}{*}{ Tobillo } & Tibia ds & 0 & 0 & 0 & 0 & 0 & 0 & 0 & 0 \\
\hline & Peroné ds & 0 & $0^{*}$ & 0 & 0 & 0 & 0 & 0 & 0 \\
\hline \multicolumn{2}{|c|}{$\mathrm{Pie}$} & $0^{*}$ & $0^{*}$ & $0^{*}$ & $0^{*}$ & $0^{*}$ & $0^{*}$ & $\mathrm{OA}$ & $\mathrm{OA}$ \\
\hline \multicolumn{2}{|c|}{$\begin{array}{l}\text { Total de regiones } \\
\text { articulares con OA }\end{array}$} & \multicolumn{2}{|c|}{1} & \multicolumn{2}{|c|}{2} & \multicolumn{2}{|c|}{2} & \multicolumn{2}{|c|}{5} \\
\hline
\end{tabular}

OM: Osteofitos marginales; P: porosidad; OS: osteofitos superficiales; ds: distal; px: proximal; A: ausencia de región anatómica/hueso, * Presencia de hasta el 50\% de la región/hueso/sup. articular. 0: ausencia de la condición.

individuo BS4, que muestra la mayor cantidad de articulaciones afectadas, corresponde al de menor edad estimada.

Debido a la desigual representación y estados de preservación de las vértebras de los cuatro individuos, sólo pudieron realizarse observaciones generales sobre artropatías espinales. En términos generales, todas sus vértebras o fragmentos observados mostraron $\mathrm{OM}$ de distinto grado de severidad en las facetas y cuerpos. En el individuo BS4, que presenta la columna completa, se observaron leves $\mathrm{OM}$ en facetas y cuerpos vertebrales de la columna cervical y toráxica y un desarrollo progresivo de OA de las facetas articulares de las lumbares y de espondilosis deformante en los cuerpos. Si bien la mayor completitud de este individuo podría explicar en parte esta "mayor" afectación comparativa, cabe recordar que se trata del individuo que mostró más regiones extra-columnares afectadas por $\mathrm{OA}$, a pesar de ser el más joven del entierro.

En cuanto a signos de traumas, ninguno de los individuos mostró fracturas ni lesiones atribuibles a algún tipo de arma. No obstante, los individuos BS1 y BS4 mostraron otro tipo de alteraciones óseas cuyo diagnóstico diferencial indicaría una etiología traumática. El individuo BS1 presentó lesiones en el cráneo y en el húmero izquierdo. En el occipital se observó un crecimiento óseo ubicado debajo de la línea nucal y con dirección subparalela a la misma. Esta excrescencia de apariencia discontinua e irregular, protruye desde la superficie externa del occipital formando una suerte de "puente óseo", de $55 \mathrm{~mm}$ de largo y una altura máxima de $20 \mathrm{~mm}$, a ambos lados de la cresta occipital externa, ligado en tres puntos a la tabla externa del hueso occipital (Fig. 3). En esta región se encuentran las inserciones para el recto posterior menor de la cabeza -que se inserta a ambos lados de la cresta occipital externa-, el semiespinal de la cabeza y el oblicuo superior. El húmero izquierdo de este individuo muestra un crecimiento óseo de características similares al hallado en el occipital, en la diáfisis media anterolateral, en la unión entre la tuberosidad deltoidea y la cresta de la tuberosidad mayor. En esta zona se inserta el músculo deltoideo. Ambas lesiones fueron radiografiadas. Sus características macroscópicas y radiológicas similares sugieren una etiología común. Entre los diagnósticos diferenciales se consideraron 


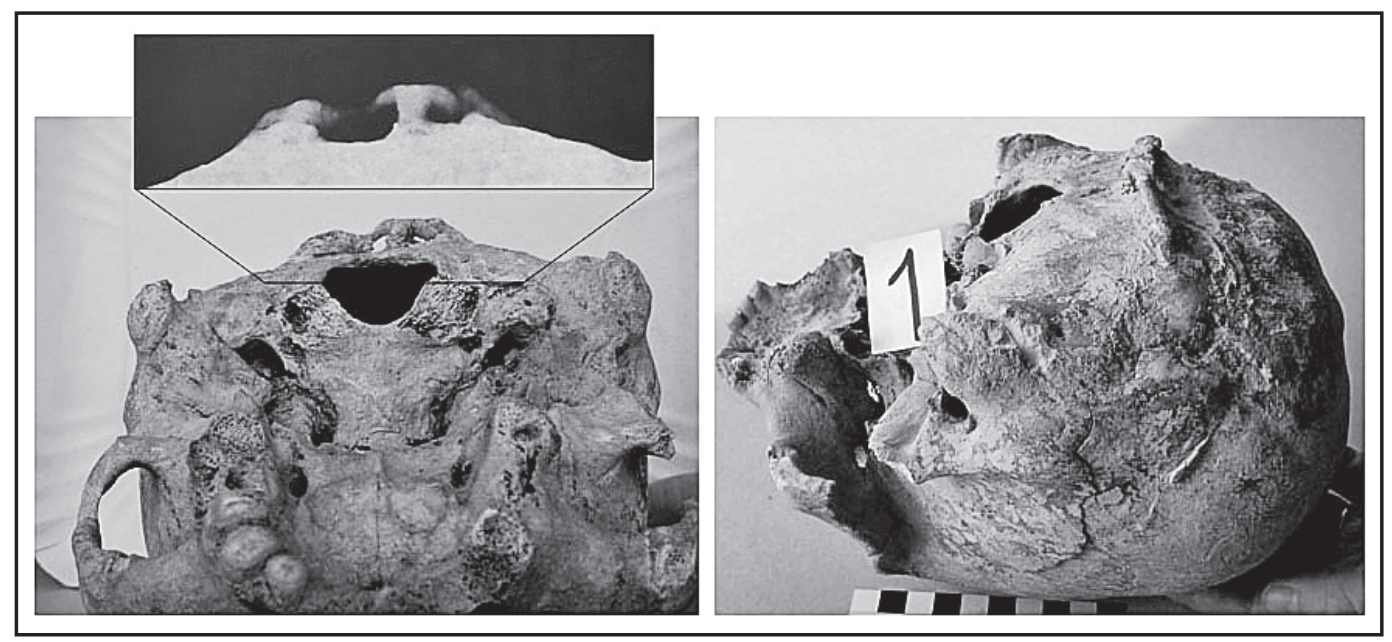

Fig. 3. Lesión occipital de BS1: norma inferior con detalle radiológico (izq.); norma lateral (der.).

la miositis osificante traumática ${ }^{2}$ y los osteocondromas $^{3}$. La morfología de ambas lesiones y su ubicación apoyan más fuertemente el diagnóstico de miositis osificante traumática. En primer lugar, la lesión del cráneo se halla en la pars squama del occipital, que se desarrolla por osificación intramembranosa y no endocondral, como requieren los osteocondromas. Asimismo, la lesión se halla en una zona de inserción muscular. Para el caso del húmero, el hecho de que la lesión no se ubique en una zona metafisiaria sino en una región de inserción muscular de la diáfisis media contribuye a descartar el diagnóstico de osteocondroma. Por último, radiológicamente ninguna de las lesiones muestra continuidad entre el tejido cortical y el esponjoso del hueso huésped y la protuberancia, otra característica diagnóstica de los osteocondromas.

2 La miositis osificante traumática resulta de la calcificación u osificación de un hematoma. Suele deberse a la avulsión de la inserción ósea de un músculo y/o tendón, lo cual genera un hematoma, que estimula al periostio a participar en su reorganización. Traumas leves pueden ocasionar miositis de este tipo (Aufderheide y Rodríguez Martín 1998, Ortner 2003).

3 Los osteocondromas son tumores benignos, que se originan en el cartílago de la placa de crecimiento de los huesos que se desarrollan por osificación endocondral. Su crecimiento es similar al del hueso endocondral huésped y se detiene cuando la placa epifisiaria más cercana se fusiona. Clínicamente suelen ser asintomáticos. Más frecuentemente afectan fémures y húmeros, aunque también se ven en escápulas, coxales, clavículas y costillas. Radiológicamente muestran continuidad del tejido cortical y esponjoso entre el hueso normal y el osteocondroma (Aufderheide y Rodríguez Martín 1998, Ortner 2003).
Por su parte, el individuo BS4 mostró una imagen osteolítica en el calcáneo izquierdo de aprox. 15 mm de diámetro y profundidad máxima, de forma irregular y bordes escleróticos, ubicada en el tubérculo calcáneo. Allí se inserta el tendón de Aquiles, correspondiente a los músculos gastrocnemius y soleus de la pantorrilla, que permiten la flexión plantar del pie. La morfología y ubicación de la lesión también sugiere un origen traumático.

Por último, se registraron osteomas en forma de botón en distintos sectores del cráneo de los individuos BS2 y BS4. Estos tumores benignos son más comunes en hombres que en mujeres y se observan con mayor frecuencia a partir de las cuarta y quinta décadas de vida (Aufderheide y RodríguezMartín 1998).

Estudio isotópico: En el marco de un conocimiento general de la ecología isotópica del área, se realizaron análisis de $\delta^{13} \mathrm{C}$ y $\delta^{15} \mathrm{~N}$ sobre colágeno de muestras óseas de cada individuo (Tabla 6) con el fin de evaluar la importancia relativa de los recursos marinos en la dieta. Los resultados indicarían dietas predominantemente marinas, de elevado nivel trófico para todos los individuos (ver Favier Dubois et al. 2009). Asimismo, aún teniendo en cuenta el pequeño tamaño de la muestra, cabe destacar que se obtuvieron valores de $\delta^{15} \mathrm{~N}$ más bajos para los individuos femeninos que para los masculinos, lo cual indicaría un nivel trófico algo menor del alimento consumido por los primeros. 
Tabla 6. Valores de isótopos de $\delta^{13} \mathrm{C}$ y $\delta^{15} \mathrm{~N}$ obtenidos para los individuos de El Buque Sur.

\begin{tabular}{|c|c|c|c|c|}
\hline $\begin{array}{c}\text { Individuo } \\
\text { (Sexo, edad) }\end{array}$ & Muestra & $\begin{array}{c}\delta^{\mathbf{1 3}} \mathrm{C} \text { USF } \\
\% 0\end{array}$ & $\begin{array}{c}\delta^{\mathbf{1}} \mathrm{N} \text { USF } \\
\% 0\end{array}$ & $\mathrm{~N}^{\circ}$ LAB (USF) \\
\hline BS1 (M, 40-44,9) & astrágalo & $-13,2$ & 17,1 & 10063 \\
\hline BS2 (F, 45-59.9) & navicular & $-12,8$ & 16,6 & 10064 \\
\hline BS3 (F,40-49.9) & navicular & $-13,1$ & 15,8 & 10065 \\
\hline BS4 (M, 25-40) & navicular & $-12,7$ & 17,4 & 10066 \\
\hline
\end{tabular}

Datos tomados de Favier Dubois et al. 2009.

\section{DISCUSIÓN Y CONCLUSIÓN}

El entierro de El Buque Sur es uno de los 21 loci con evidencia de inhumaciones registrados hasta el momento en la costa rionegrina del golfo San Matías, donde se ha recuperado un número mínimo de 53 individuos (Favier Dubois et al. 2007 y Mariano 2009). En este marco, el entierro constituye un hallazgo de características particulares, ya que un importante porcentaje del registro bioarqueológico del área se caracteriza por provenir de contextos superficiales muy impactados o de colecciones de particulares. En ambos casos, ya sea por las malas condiciones de preservación (Borella et al. 2007) o por la pérdida de importante información contextual (Favier Dubois et al. 2007; Mariano 2009), se dificulta la caracterización de los contextos mortuorios y la realización de estudios específicos, como por ejemplo los paleopatológicos. En este contexto, el hallazgo de El Buque Sur posibilitó un abordaje metodológico muy diferente al de los estudios que venían realizándose (Borella et al. 2007). Luego del tratamiento en el laboratorio, se observó que los esqueletos mostraban un grado de completitud suficiente para determinar la composición del entierro, abordar estudios paleopatológicos y realizar análisis isotópicos con información precisa sobre el sexo y la edad de los individuos muestreados. Asimismo, los estudios tafonómicos efectuados y la posibilidad de recuperar in situ uno de los individuos permitieron estudiar aspectos vinculados con el tratamiento mortuorio que, hasta el momento del hallazgo, sólo habían podido abordarse de forma indirecta, a través de la lectura de los escasos antecedentes disponibles para el área o de información proporcionada por los lugareños. En este sentido, hasta la fecha El Buque Sur es el único entierro primario múltiple recuperado con metodología arqueológica y ha servido de base para sustentar lo inferido sobre el tipo de entierro original de otros conjuntos osteológicos superficiales que mostraban mayor daño tafonómico (e.g. Borella et al. 2007).

Para ubicar el caso del Buque Sur en el marco de la estructura del registro bioarqueológico del golfo San Matías, conviene detenerse en las tendencias principales observadas en los estudios bioarqueológicos de escala areal realizados hasta el momento (Favier Dubois et al. 2007, 2008; Mariano 2009). En primer lugar, se ha observado una mayor cantidad y densidad de entierros humanos en la costa norte del golfo. Si se incluye el sector septentrional de la costa oeste, (donde se encuentra BS), el $95 \%$ de los loci con inhumaciones se hallan en este sector del litoral. Como se ha mencionado, esta distribución diferencial se observa también en el resto de los componentes del registro arqueológico. En segundo lugar, las 21 dataciones obtenidas sobre restos humanos (Favier Dubois et al. 2009) permiten distinguir dos grupos de fechados: uno más temprano, que abarca entre ca. 3100 y 2200 años AP y otro más reciente, entre ca. 1500 y 420 años AP -con un hiato entre ellos, que podría deberse a cuestiones de muestreo. Estos bloques cronológicos van aparejados a cambios en la señal isotópica de los restos y parecen mostrar también un correlato en términos de las modalidades funerarias representadas. En el período más antiguo, donde se incluye BS con ca. 2300 años AP, sólo se registran entierros primarios de individuos flexionados, ubicados en dunas, sin estructuras obstrusivas ni artefactos asociados. En el segundo periodo, coexisten entierros primarios y secundarios de distintas características, que evidencian mayor variabilidad en las formas de tratamiento mortuorio (Mariano 2009).

Específicamente en relación con las diferencias en las señales isotópicas entre los dos periodos, cabe mencionar que los individuos de El Buque Sur corresponden al período más temprano, en el que los valores de $\delta^{13} \mathrm{C}$ y $\delta^{15} \mathrm{~N}$ sugieren una dieta predominantemente marina, de elevado nivel trófico. En 
cambio, para el periodo más tardío la señal isotópica es consistente con dietas mixtas a continentales, de menor nivel trófico en promedio. Favier Dubois et al. (2009) han vinculado estas tendencias temporales -observables no sólo en el registro isotópico, sino también en otros componentes del registro arqueológico, como el arqueofaunístico y el tecnológico- con modalidades distintas de uso del litoral rionegrino durante el Holoceno medio y tardío. En su modelo, el entierro de El Buque Sur correspondería a un momento de uso intensivo del espacio costero y los recursos marinos (peces, mamíferos marinos, moluscos, crustáceos y aves) vinculado con tecnologías tales como la pesca con redes. En el período más tardío se verifica un cambio hacia un uso moderado de la costa, en el que se agregan al registro arqueológico especies animales terrestres (guanaco, rheidos y pequeños mamíferos) y tecnologías nuevas, como la cerámica y las puntas de proyectil pequeñas (referibles al uso del arco y flecha), al tiempo que va disminuyendo la frecuencia de los artefactos vinculados con la pesca (Favier Dubois et al. 2009). Por último, el estudio paleopatológico efectuado constituye el primer análisis sobre restos óseos contextualizados espacial y temporalmente en el área. Por el momento, el tamaño de la muestra no permite realizar mayores consideraciones cuantitativas sobre la prevalencia de las distintas condiciones paleopatológicas registradas. No obstante, los datos obtenidos son el primer paso para conocer el rango de variabilidad en términos de salud de las poblaciones cazadorasrecolectoras de las cuales deriva esta muestra. Así, atendiendo a un patrón usualmente observado en los estudios paleopatológicos sobre esqueletos adultos, las condiciones patológicas más generalizadas se observaron a nivel dental y articular. Como hemos señalado, los indicadores de salud y desgaste dental se presentan consistentes con los patrones observados en otras muestras de cazadores-recolectores de ambientes relativamente áridos del país, donde la dieta abrasiva (a su vez preparada/consumida en ambientes abrasivos) y la relativamente escasa disponibilidad de alimentos vegetales cariogénicos, favorecen bajas frecuencias de caries, altos grados de desgaste, presencia de enfermedad pulpoalveolar (i.e. los abscesos) causada por el desgaste $y$, en ocasiones, la consecuente pérdida antemortem de piezas dentales (e.g. Bernal et al. 2007;
L'Heureux 2002; Novellino y Guichón 1997-1998).

La distribución generalizada de osteoartrosis y los casos de osteomas hallados, son consistentes con la edad estimada para los individuos de la muestra, aunque se destaca la mayor severidad de OA en el individuo más joven, ratificando la etiología multifactorial de esta condición (Weiss y Jurmain 2007). Por su parte, la probable miositis osificante traumática se presenta como un caso particular de respuesta a estrés traumático, cuya prevalencia en estas poblaciones deberá ser evaluada en el futuro, al aumentar el tamaño muestral.

En suma, el hallazgo de El Buque Sur ha permitido obtener información bioarqueológica a partir del estudio de un entierro de características únicas para el área hasta la fecha. Nuevas investigaciones permitirán evaluar las tendencias esbozadas y mejorar nuestra comprensión de las formas del uso del espacio costero, la dieta, la salud y las prácticas mortuorias de las poblaciones que habitaron el litoral rionegrino durante el Holoceno medio y tardío.

\section{AGRADECIMIENTOS}

A la comunidad mapuche local, al Dr. L. Bosio, Dra. F. Borella, F. Scartascini, Lic. S. Lanzellotti, C. Mora, S. Plos, A. Granosky, familia Tarruella y Lic. T. Civalero por su colaboración en diferentes aspectos de este trabajo. Las investigaciones fueron financiadas por los Proyectos PIP CONICET 6415 y PICT Agencia 38264.

\section{BIBLIOGRAFÍA CITADA}

AUFDERHEIDE, A. y C. RODRÍGUEZ-MARTíN. 1998. The Cambridge Encyclopedia of Human Paleopathology. Cambridge University Press. Cambridge.

BERNAL, V., P. NOVELLINO, P. GONZÁLEZ y S. PEREZ. 2007. Role of wild plant foods among late Holocene hunter-gatherers from Central and North Patagonia (South America): An approach from dental evidence. American Journal of Physical Anthropology 133: 1047-1059.

BORELLA, F. y C. FAVIER DUBOIS. 2005. Primeros resultados del proyecto arqueológico en la costa del golfo de San Matías (Pcia. de Río Negro) En INCUAPA 10 años. Perspectivas Contemporáneas en la Arqueología Pampeana. Editado por G. Politis. Serie Monográfica del INCUAPA N ${ }^{\circ}$. FACSO-UNICEN. En prensa. 
BORELLA. F., C. FAVIER DUBOIS y L. MANZI. 2006. The exploitation of coastal resources and space use patterns in norpatagonian coast during Late Holocene times. Actas del ICAZ $10^{\text {th }}$ Conference, México, pp. 36-37.

BORELLA, F., C. MARIANO y C. FAVIER DUBOIS. 2007. Procesos tafonómicos en restos humanos de la localidad arqueológica de Bajo de la Quinta, Golfo San Matías (Río Negro). En: Arqueología de Fuego-Patagonia. Levantando piedras, desenterrando huesos... y develando arcanos; pp.: 403-410. Editores: F. Morello, M. Martinic, A Prieto y G. Bahamonde. Ediciones CEQUA. Punta Arenas.

BÓRMIDA, M. 1953-54. Los Antiguos Patagones. Estudio de Craneología. Runa VI: 5-96.

BROOKS, S. y J. SUCHEY. 1990. Skeketal age determination based the os pubis: A comparison of the Acsádi-Nemeskéri and Suchey-Brooks methods. Human Evolution 5: 227-238.

BUIKSTRA, J. y D. UBELAKER. 1994. Standards for data collection from human skeletal remains. Arkansas Archaeological Survey Research Series 44.

CLARKE, N. y R. HIRSCH. 1991. Tooth Dislocation: The relationship with Tooth Wear and Dental Abscesses. American Journal of Physical Anthropology 85: 293-298.

FAVIER DUBOIS, C., F. BORELLA, L. MANZI, M. CARDILLO, S. LANZELOTTI, F. SCARTASCINI, C. MARIANO y E. BORGES VAZ. 2006. Aproximación regional al registro arqueológico de la costa rionegrina. En: Arqueología de la Costa Patagónica. Perspectivas para la conservación. Editoras I. Cruz y M. S. Caracotche. Capítulo 3, pp. 50-68. Universidad de la Patagonia Austral, Río Gallegos

FAVIER DUBOIS, C., F. BORELLA y R. TYKOT. 2009. Explorando tendencias en el uso humano del espacio y los recursos en el litoral rionegrino durante el Holoceno tardío y medio. En: Arqueología de Patagonia: una mirada desde el último confín. Tomo 2; pp. 985-998. Compilado por M. Salemme, F. Santiago, M. Alvarez, E. Piana, M. Vázquez y E. Mansur. Editorial Utopías. Ushuaia.

FAVIER DUBOIS, C., S. GARCÍA GURAIEB, F. BORELLA y C. MARIANO, C. 2007. Primeros avances acerca del registro bioarqueológico de la costa rionegrina. Resúmenes expandidos del XVI Congreso Nacional de Arqueología Argentina, Jujuy, tomo III, pp. 359-364.

GARCÍA GURAIEB, S. 2006. Salud y Enfermedad en cazadoresrecolectores del Holoceno-tardío en la cuenca del lago Salitroso (Santa Cruz). Intersecciones en Antropología 7: $37-48$.
HILLSON, S. 1996. Dental Anthropology . Cambridge University Press. Cambridge.

L'HEUREUX, G. L. 2002. Inferencias paleodietarias a partir del análisis de los patrones de desgaste dental y frecuencia de caries en muestras de restos humanos del Holoceno del Sudeste de la Región Pampeana. En: Del Mar a los Salitrales. Diez mil años de Historia Pampeana en el Umbral del Tercer Milenio. Editado por D. L. Mazzanti, M. A. Berón, F. W. Oliva, pp. 127-140. Facultad de Humanidades. Universidad Nacional de Mar del Plata.

LOVEJOY, O., R. MEINDL, T. PRYZBECK, y R. MENSFORTH. 1985. Chronological methamorphosis of the auricular surface of the ilium: A new method for the determination of adult skeletal age at death. American Journal of Physical Anthropology 68: 15-28.

LOVELL, N. 2000. Paleopathological description and diagnosis. En: Biological Anthropology of the human skeleton. Editores: M. Katzenberg y S. Saunders, pp. 217-248. Willey-Liss Inc.

MARIANO, C. 2009. Prácticas mortuorias y registro bioarqueológico en la costa rionegrina del Golfo San Matías. Tesis de licenciatura. Universidad Nacional del Centro de la Provincia de Buenos Aires, Facultad de Ciencias Sociales, Olavarría. MS.

MENSFORTH, R. 1991. Paleoepidemiology of porotic hyperostosis in the Libben and BT-5 skeletal populations. Kirtlandia 46: 1-47.

MONDINI, M. 2003. Formación del registro arqueofaunístico en abrigos rocosos de la Puna argentina. Tafonomía de carnívoros. Tesis Doctoral. Facultad de Filosofía y Letras, Universidad de Buenos Aires. Buenos Aires. MS.

NOVELLINO, P. y R. GUICHÓN. 1997-1998. Comparación de indicadores de dieta y salud entre el Sur de Mendoza y el Sur de San Juan-Norte de Mendoza. Relaciones de la Sociedad Argentina de Antropología XXII-XXII: 125-138.

ORTNER, D. 2003. Identification of Human Skeleton Conditions in Human Skeletal Remains. Smithsonian Institution. National Museum of Natural History. Academic Press.

ROGERS, J. y T. WALDRON. 1995. A field guide to joint disease in archaeology. John Wiley \& Sons.

SÁNCHEZ ALBORNOZ, N. 1967. Hachas y placas de San Antonio Este (Río Negro). Runa, Vol. X, 1-2: 455-465.

STUIVER, M. y P. J. REIMER. 1993. Extended 14C data base and revised CALIB 3.0 14C age calibration program. Radiocarbon, 35:215-230.

WEISS, E. y R. JURMAIN. 2007. Osteoarthritis Revisited: A Contemporary Review of Aetiology. International Journal of Osteoarchaeology 17: 437-450. 
WESTON, D. 2008. Investigating the specificity of periosteal reactions in pathology museum specimens. American Journal of Physical Anthropology 137(1): 48-59.
WHITE, T. D. y P. A. FOLKENS. 2005. The Human Bone Manual. Elsevier Academic Press. San Diego. 